\title{
TWO-BOUNDARY PROBLEMS FOR A RANDOM WALK WITH NEGATIVE GEOMETRIC JUMPS
}

\author{
T. V. KADANKOVA
}

\begin{abstract}
Two-boundary problems for a random walk with negative geometric jumps are considered, and the corresponding results for a usual semicontinuous random walk are generalized for them. The following results are obtained: the probability distribution of ruin is found and expressed in terms of the lower and upper boundaries; formulas are given for the joint distribution of the infimum, supremum, and the walk itself at an arbitrary time instance; the transient probabilities and ergodic distribution are evaluated for the process describing the evolution of the random walk with two boundaries.
\end{abstract}

Two-boundary problems for random walks and stochastic processes have several applications in the queue theory, storage and inventory theories, reliability theory, and in many other fields.

Two-boundary problems have been studied for semicontinuous random walks and for semicontinuous stochastic processes. Several methods are known for solving those problems, namely combinatorial [1], resolvent [2]-[6], factorization [7], and renewal [8] methods.

In this paper we solve two-boundary problems for random walks with negative geometric jumps. This model is a generalization of a usual model of semicontinuous random walks.

\section{Main Notation}

Let $\alpha \in\{0,1, \ldots\}$ be a nonnegative integer-valued random variable,

$$
\mathrm{E}\left[\theta^{\alpha}\right]=\sum_{i=0}^{\infty} a_{i} \theta^{i}, \quad a_{i}=\mathrm{P}[\alpha=i], \quad \mathrm{P}[\alpha>1]>0, \quad|\theta| \leq 1 .
$$

Consider the random variable

$$
\xi=\alpha-\beta, \quad \xi \in\{0, \pm 1, \ldots\}=\mathbf{Z},
$$

where $\beta \in\{1,2, \ldots\}$ is a positive integer-valued random variable distributed geometrically with parameter $b \in[0,1)$, that is,

$$
\mathrm{P}[\beta=n]=(1-b) b^{n-1}, \quad n>0 .
$$

It is clear that

$$
\mathrm{E}\left[\theta^{\xi}\right]=\mathrm{E}\left[\theta^{\alpha}\right] \frac{(1-b) / \theta}{1-b / \theta} \stackrel{\text { def }}{=} \sum_{i=-\infty}^{\infty} p_{i} \theta^{i}, \quad|\theta|=1 .
$$

An easy evaluation yields the distribution of the random variable $\xi$ :

$$
p_{i}=\mathrm{P}[\xi=i]=(1-b) b^{-i-1} \mathrm{E}\left[b^{\alpha} ; \alpha>i\right], \quad i \in \mathbf{Z} .
$$

2000 Mathematics Subject Classification. Primary 60G50, 60J50. 
Some of the properties of the distribution $p_{i}, i \in \mathbf{Z}$, are listed in the following result.

Lemma 1. For all $i \in \mathbf{Z}$,

$$
\begin{gathered}
b p_{i}-p_{i-1}= \begin{cases}0, & i<0, \\
-(1-b) a_{i}, & i \geq 0,\end{cases} \\
b \mathrm{P}[\xi \geq i]-\mathrm{P}[\xi \geq i-1]=-(1-b) \mathrm{P}[\alpha \geq i], \\
b \mathrm{P}[\xi \leq i]-\mathrm{P}[\xi \leq i-1]=-(1-b) \mathrm{P}[\alpha \leq i],
\end{gathered}
$$

where $\mathrm{P}[\alpha=m]=0$ for $m<0$.

These equalities follow from the explicit expression (1) for the distribution of the random variable $\xi$.

Let $\left\{\xi, \xi_{i}^{\prime}\right\}, i>0$, be a sequence of independent identically distributed random variables and let $\xi_{n}, n \geq 0$, be a random walk such that

$$
\xi_{0}=0, \quad \xi_{n}=\xi_{1}^{\prime}+\cdots+\xi_{n}^{\prime}, \quad n>0 .
$$

The moment generating function of increments of the random walk $\xi_{n}, n \geq 0$, satisfies

$$
\sum_{n=0}^{\infty} t^{n} \mathrm{E}\left[\theta^{\xi_{n}}\right]=\frac{1}{1-t \mathrm{E}\left[\theta^{\xi}\right]}=\left\{1-t \mathrm{E}\left[\theta^{\alpha}\right] \frac{(1-b) / \theta}{1-b / \theta}\right\}^{-1}, \quad|\theta|=1, t \in[0,1) .
$$

The equation

$$
1-\frac{b}{\theta}-(1-b) \frac{t}{\theta} \mathrm{E}\left[\theta^{\alpha}\right]=0
$$

has a unique root $\theta=c(t)$ in the domain $|\theta|<1$. Moreover the root is positive and $b<c(t)<1$. This result is well known for semicontinuous random walks [9]; the proof for the case under consideration is similar.

Definition 1. A sequence $R_{k}(t), k>0$, is called the resolvent of the random walk $\xi_{n}$, $n \geq 0$, if

$$
R(\theta, t)=\sum_{k=1}^{\infty} \theta^{k} R_{k}(t)=\left[\frac{b}{\theta}+(1-b) \frac{t}{\theta} \mathrm{E}\left[\theta^{\alpha}\right]-1\right]^{-1}, \quad|\theta|<c(t) .
$$

Since the right-hand side of (3) is analytic for $|\theta|<c(t)$, it can be expanded in a power series. In what follows we need the first term of the sequence $R_{k}(t), k>0$ :

$$
R_{1}(t)=(b+(1-b) t \mathrm{P}[\alpha=0])^{-1} .
$$

Definition 2. A sequence $R_{k}, k>0$, is called the potential of the random walk $\xi_{n}$, $n \geq 0$, if

$$
R(\theta)=\sum_{k=1}^{\infty} \theta^{k} R_{k}=\left[\frac{b}{\theta}+(1-b) \frac{1}{\theta} \mathrm{E}\left[\theta^{\alpha}\right]-1\right]^{-1}, \quad|\theta|<c(1) \leq 1 .
$$

The following two equalities are used later when solving two-boundary problems.

Lemma 2. For all $k>0$,

$$
\begin{gathered}
R_{k}(t)+t(1-b) b^{-k-1} \sum_{i=1}^{k} R_{i}(t) b^{i} \mathrm{E}\left[b^{\alpha} ; \alpha \leq k-i\right]=b^{-k}, \\
S_{k}(t)+t(1-b) b^{-k-1} \sum_{i=1}^{k} S_{i}(t) b^{i} \mathrm{E}\left[b^{\alpha} ; \alpha \leq k-i\right]=\frac{1}{1-b}\left[b^{-k}-1\right],
\end{gathered}
$$


where

$$
S_{k}(t)=\sum_{i=1}^{k} R_{i}(t)
$$

Proof. Denote the left-hand side of (5) by $A_{k}, k>0$. Then

$$
\sum_{k=1}^{\infty} \theta^{k} A_{k}=R(\theta, t)+t(1-b) \frac{1}{b} R(\theta, t) \frac{\mathrm{E}\left[\theta^{\alpha}\right]}{1-\theta / b}, \quad|\theta|<b .
$$

It follows from the definition of the resolvent (3) that

$$
R(\theta, t)\left[\frac{b}{\theta}+(1-b) \frac{t}{b} \mathrm{E}\left[\theta^{\alpha}\right]-1\right]=1, \quad|\theta|<c(t),
$$

whence

$$
\sum_{k=1}^{\infty} \theta^{k} A_{k}=\frac{\theta}{b}\left\{1-\frac{\theta}{b}\right\}^{-1}, \quad|\theta|<b
$$

Comparing the coefficients for $\theta^{k}, k>0$, on both sides of the latter equality, we get $A_{k}=b^{-k}, k>0$. Equality (6) is proved similarly.

Now we turn to solving the two-boundary problems.

\section{Ruin PROBLEM}

Fix an integer $N>1$ and put $\mathrm{P}\left[\xi_{0}=k\right]=1, k \in\{1, \ldots, N-1\}$. Let

$$
\tau_{k}=\inf \left\{n>0: \xi_{n} \notin\{1, \ldots, N-1\}\right\}
$$

be the first moment when the random walk $\xi_{n}, n \geq 0$, exits the set $\{1, \ldots, N-1\}$. We also introduce the first moments when the random walk $\xi_{n}, n \geq 0$, exits the set $\{1, \ldots, N-1\}$ through the upper and lower boundary, respectively:

$$
\begin{aligned}
& \tau_{k}^{+}=\inf \left\{n>0: \xi_{n} \notin\{1, \ldots, N-1\}, \xi_{\tau_{k}} \geq N\right\}, \\
& \tau_{k}^{-}=\inf \left\{n>0: \xi_{n} \notin\{1, \ldots, N-1\}, \xi_{\tau_{k}} \leq 0\right\} .
\end{aligned}
$$

Theorem 1. Let

$$
\mathrm{E}\left[t^{\tau_{k}^{-}}\right]=\mathrm{E}\left[t^{\tau_{k}} ; \xi_{\tau_{k}} \leq 0\right], \quad \mathrm{E}\left[t^{\tau_{k}^{+}}\right]=\mathrm{E}\left[t^{\tau_{k}} ; \xi_{\tau_{k}} \geq N\right]
$$

be the moment generating functions of the "ruin" times $\tau_{k}^{-}$and $\tau_{k}^{+}, k \in\{1, \ldots, N-1\}$. Then

$$
\begin{gathered}
\mathrm{E}\left[t^{\tau_{k}^{-}}\right]=\frac{b^{N}}{1-b} \frac{R_{N-k}(t)}{\hat{R}_{N}(b, t)} \\
\mathrm{E}\left[t^{\tau_{k}^{+}}\right]=1-\frac{R_{N-k}(t)}{\hat{R}_{N}(b, t)}\left[\frac{b^{N}}{1-b}+(1-b)(1-t) \hat{S}_{N}(b, t)\right]+(1-b)(1-t) S_{N-k}(t),
\end{gathered}
$$

where $R_{k}(t), k>0$, is the resolvent (3) of the random walk $\xi_{n}, n \geq 0$,

$$
\hat{R}_{N}(b, t)=\sum_{i=N}^{\infty} b^{i} R_{i}(t), \quad S_{k}(t)=\sum_{i=1}^{k} R_{i}(t), \quad \hat{S}_{N}(b, t)=\sum_{i=N}^{\infty} b^{i} S_{i}(t) .
$$

Corollary 1. Let

$$
\mathrm{P}_{k}^{-}=\mathrm{P}\left[\xi_{\tau_{k}} \leq 0\right] \quad \text { and } \quad \mathrm{P}_{k}^{+}=\mathrm{P}\left[\xi_{\tau_{k}} \geq N\right]
$$


be the "ruin" probabilities corresponding to the lower and upper boundaries, respectively. Denote by $\mathrm{E}\left[\tau_{k}\right]$ the mean "ruin" time. Then

$$
\begin{gathered}
\mathrm{P}_{k}^{-}=\frac{b^{N}}{1-b} \frac{R_{N-k}}{\hat{R}_{N}(b)}, \quad \mathrm{P}_{k}^{+}=1-\frac{b^{N}}{1-b} \frac{R_{N-k}}{\hat{R}_{N}(b)}=1-\mathrm{P}_{k}^{-}, \\
\mathrm{E}\left[\tau_{k}\right]=b^{N} \frac{R_{N-k}}{\hat{R}_{N}(b)} S_{N-1}+R_{N-k}-(1-b) S_{N-k},
\end{gathered}
$$

where $R_{k}, k>0$, is the potential of the random walk $\xi_{n}, n \geq 0$,

$$
\hat{R}_{N}(b)=\sum_{i=N}^{\infty} b^{i} R_{i}, \quad S_{k}=\sum_{i=1}^{k} R_{i}
$$

Proof of Theorem 1. Let $\mathrm{P}\left[\xi_{0}=N-k\right]=1, k \in\{1, \ldots, N-1\}$. By the full probability formula, the functions

$$
\varphi_{k}(t)=\mathrm{E}\left[t^{\tau_{N-k}^{-}}\right], \quad k \in\{1, \ldots, N-1\}, t \in[0,1),
$$

satisfy the following system of equations:

$$
\varphi_{k}(t)=t \mathrm{P}[\xi \leq k-N]+t \sum_{i=1}^{N-1} \varphi_{i}(t) p_{k-i}, \quad k \in\{1, \ldots, N-1\} .
$$

System (8) is regular, that is,

$$
t \sum_{i=1}^{N-1} p_{k-i}<1-t b^{N-2} \mathrm{E}\left[b^{\alpha}\right] .
$$

Therefore it has a unique solution (see [10]). We are going to find this solution in an explicit form. When solving this problem we follow a method that was useful for solving other two-boundary problems.

The functions $b \varphi_{k+1}(t)$ are such that

$$
b \varphi_{k+1}(t)=t b \mathrm{P}[\xi \leq k+1-N]+t b \sum_{i=1}^{N-1} \varphi_{i}(t) p_{k+1-i}, \quad k \in\{0, \ldots, N-2\} .
$$

Subtracting this equation from (8) and applying (2) we get

$$
b \varphi_{k+1}(t)-\varphi_{k}(t)=-t(1-b) \sum_{i=1}^{k+1} \varphi_{i}(t) a_{k+1-i}, \quad k \in\{1, \ldots, N-2\} .
$$

Consider equation (9) for all $k \geq 1$ :

$$
b \varphi_{k+1}(t)-\varphi_{k}(t)=-t(1-b) \sum_{i=1}^{k+1} \varphi_{i}(t) a_{k+1-i}, \quad k \geq 1,
$$

and solve it by using the moment generating function method. Put

$$
\Phi(\theta, t)=\sum_{k=1}^{\infty} \theta^{k} \varphi_{k}(t), \quad|\theta|<c(t) .
$$

Rewriting equation (10) for the corresponding moment generating functions we obtain

$$
\Phi(\theta, t)\left(\frac{b}{\theta}+(1-b) \frac{t}{\theta} \mathrm{E}\left[\theta^{\alpha}\right]-1\right)=\varphi_{1}(t) \frac{1}{R_{1}(t)}, \quad|\theta|<c(t) .
$$


By the definition of the resolvent (3) we get from the latter equation that

$$
\varphi_{k}(t)=\varphi_{1}(t) \frac{R_{k}(t)}{R_{1}(t)}, \quad k \geq 1 .
$$

The function $\varphi_{k}(t)$ in (11) satisfies equation (10) for all $k \geq 1$, thus it is a solution of equation (9) for $k \in\{1, \ldots, N-2\}$. A solution of initial equation (8) belongs to the set of solutions of equation (9) for which the function $\varphi_{1}(t)$ is a free parameter. It remains to find a function $\varphi_{1}^{*}(t)$ such that $\varphi_{k}(t)$ defined by (11) satisfies equation (8). Substituting the function $\varphi_{k}(t)$ from (11) into the initial equation (8) and using Lemma 2 we get

$$
\varphi_{1}^{*}(t)=\frac{b^{N}}{1-b} \frac{R_{1}(t)}{\hat{R}_{N}(b, t)}, \quad \hat{R}_{N}(b, t)=\sum_{i=N}^{\infty} R_{i}(t) b^{i} .
$$

Thus

$$
\varphi_{k}(t)=\mathrm{E}\left[t^{\tau_{N-k}^{-}}\right]=\frac{b^{N}}{1-b} \frac{R_{k}(t)}{\hat{R}_{N}(b, t)}, \quad k \in\{1, \ldots, N-1\},
$$

and the first equality of Theorem 1 is proved.

Now we prove the second equality. Let $\mathrm{P}\left[\xi_{0}=N-k\right]=1, k \in\{1, \ldots, N-1\}$. By the full probability formula, the functions

$$
\psi_{k}(t)=\mathrm{E}\left[t^{\tau_{N-k}} ; \xi_{\tau_{N-k}} \geq N\right], \quad t \in[0,1),
$$

satisfy the system of equations

$$
\psi_{k}(t)=t \mathrm{P}[\xi \geq k]+t \sum_{i=1}^{N-1} \psi_{i}(t) p_{k-i}, \quad k \in\{1, \ldots, N-1\} .
$$

The equation for $b \psi_{k+1}(t)$ is given by

$$
b \psi_{k+1}(t)=t b \mathrm{P}[\xi \geq k+1]+t b \sum_{i=1}^{N-1} \psi_{i}(t) p_{k+1-i}, \quad k \in\{0, \ldots, N-2\} .
$$

Subtracting this equation from (12) we obtain that for all $k \geq 1$,

$$
b \psi_{k+1}(t)-\psi_{k}(t)=-t(1-b) \sum_{i=1}^{k+1} \psi_{i}(t) a_{k+1-i}-t(1-b) \mathrm{P}[\alpha \geq k+1], \quad k \geq 1 .
$$

Put

$$
\Psi(\theta, t)=\sum_{k=1}^{\infty} \theta^{k} \psi_{k}(t), \quad|\theta|<c(t) .
$$

Rewriting equation (13) for the corresponding moment generating functions and using the definition of the resolvent (3) we get

$$
\Psi(\theta, t)=\frac{\theta}{1-\theta}+\frac{\psi_{1}(t)-1}{R_{1}(t)} R(\theta, t)+(1-b)(1-t) \frac{\theta}{1-\theta} R(\theta, t), \quad|\theta|<c(t) .
$$

Comparing the coefficients for $\theta^{k}, k>0$, on both sides of the latter equality we obtain

$$
\psi_{k}(t)=1+\frac{\psi_{1}(t)-1}{R_{1}(t)} R_{k}(t)+(1-b)(1-t) S_{k-1}(t), \quad k \geq 1 .
$$

To find a function $\psi_{1}^{*}(t)$ such that $\psi_{k}(t)$ defined by (14) satisfies the initial system of equations (12), we substitute the right-hand side of (14) into the system of equations (12) and apply Lemma 2. Then we obtain the following relation:

$$
\frac{\psi_{1}^{*}(t)-1}{R_{1}(t)}=-\frac{1}{\hat{R}_{N}(b, t)}\left[\frac{b^{N}}{1-b}+(1-b)(1-t) \hat{S}_{N}(b, t)\right]+(1-b)(1-t),
$$


where

$$
\hat{R}_{N}(b, t)=\sum_{i=N}^{\infty} b^{i} R_{i}(t), \quad \hat{S}_{N}(b, t)=\sum_{i=N}^{\infty} b^{i} S_{i}(t) .
$$

Therefore

$$
\begin{gathered}
\psi_{k}(t)=1-\frac{R_{k}(t)}{\hat{R}_{N}(b, t)}\left[\frac{b^{N}}{1-b}+(1-b)(1-t) \hat{S}_{N}(b, t)\right]+(1-b)(1-t) S_{k}(t), \\
k \in\{1, \ldots, N-1\},
\end{gathered}
$$

and the second equality of Theorem 1 is proved.

Further

$$
\sum_{n=0}^{\infty} t^{n} \mathrm{P}\left[\tau_{N-k}>n\right]=\frac{1-\varphi_{k}(t)-\psi_{k}(t)}{1-t}=\frac{(1-b) \hat{S}_{N}(b, t)}{\hat{R}_{N}(b, t)} R_{k}(t)-(1-b) S_{k}(t) .
$$

Since

$$
(1-b) \hat{S}_{N}(b, t)=\hat{R}_{N}(b, t)+b^{N} S_{N-1}(t),
$$

we have

$$
\sum_{n=0}^{\infty} t^{n} \mathrm{P}\left[\tau_{N-k}>n\right]=b^{N} \frac{R_{k}(t)}{\hat{R}_{N}(b, t)} S_{N-1}(t)+R_{k}(t)-(1-b) S_{k}(t),
$$

whence equality (7) follows. Corollary 1 follows from Theorem 1 for $t=1$.

3. The Joint Distribution of $\inf \left\{\xi_{0}, \ldots, \xi_{n}\right\}, \sup \left\{\xi_{0}, \ldots, \xi_{n}\right\}$, ANd $\xi_{n}, n \geq 0$

Assume that $\mathrm{P}\left[\xi_{0}=0\right]=1$ and consider the random variables

$$
\mu_{n}^{+}=\sup \left\{\xi_{0}, \ldots, \xi_{n}\right\}, \quad \mu_{n}^{-}=\inf \left\{\xi_{0}, \ldots, \xi_{n}\right\} .
$$

Let $k$ and $r$ be positive integers, $R_{m}(t)=0, m \leq 0$.

Theorem 2. The joint distribution

$$
\mathrm{P}_{(-r, k)}^{n}[i]=\mathrm{P}\left[-r<\mu_{n}^{-}, \xi_{n}=i, \mu_{n}^{+}<k\right], \quad-r<i<k,
$$

of the random variables $\mu_{n}^{-}, \xi_{n}$, and $\mu_{n}^{+}$satisfies

$$
\sum_{n=0}^{\infty} t^{n} \mathrm{P}_{(-r, k)}^{n}[i]=b^{N} \frac{R_{k}(t)}{\hat{R}_{N}(b, t)} R_{i+r}(t)+b R_{i+1}(t)-R_{i}(t), \quad N=k+r .
$$

Proof of Theorem 2. Fix $N>1$ and assume that $\mathrm{P}\left[\xi_{0}=N-k\right]=1, k \in\{1, \ldots, N-1\}$. Let

$$
\tau_{N-k}=\inf \left\{n>0: \xi_{n} \notin\{1, \ldots, N-1\}\right\}
$$

be the first moment when the random walk $\xi_{n}, n \geq 0$, exits the set $\{1, \ldots, N-1\}$. Denote by

$$
Q_{k, d}(n)=\mathrm{P}\left[\xi_{n}=N-d, \tau_{N-k}>n\right], \quad d \in\{1, \ldots, N-1\},
$$

the transient probability of the random walk $\xi_{n}, n>0$, on the interval $0 \leq n<\tau_{N-k}$.

By the full probability formula, the moment generating functions

$$
Q_{k, d}^{t}=\sum_{n=0}^{\infty} t^{n} Q_{k, d}(n), \quad k, d \in\{1, \ldots, N-1\}, t \in[0,1)
$$

satisfy the system of equations

$$
Q_{k, d}^{t}=\delta_{k, d}+t \sum_{i=1}^{N-1} p_{k-i} Q_{i, d}^{t}, \quad k \in\{1, \ldots, N-1\}
$$


where $\delta_{k, r}$ is the Kronecker symbol. The corresponding equation for $b Q_{k+1, d}^{t}$ is

$$
b Q_{k+1, d}^{t}=b \delta_{k+1, d}+t b \sum_{i=1}^{N-1} p_{k+1-i} Q_{i, d}^{t}, \quad k \in\{0, \ldots, N-2\} .
$$

Subtracting this equation from (15) we obtain that for all $k>0$,

$$
b Q_{k+1, d}^{t}-Q_{k, d}^{t}=b \delta_{k+1, d}-\delta_{k, d}-t(1-b) \sum_{i=1}^{k+1} a_{k+1-i} Q_{i, d}^{t} .
$$

Consider the moment generating function

$$
Q_{d}^{t}(\theta)=\sum_{k=1}^{\infty} \theta^{k} Q_{k, d}^{t}, \quad|\theta|<c(t) .
$$

Rewriting (16) for the corresponding moment generating functions we obtain

$$
Q_{d}^{t}(\theta)=\left(\frac{Q_{1, d}^{t}}{R_{1}(t)}-b \delta_{1, d}\right) R(\theta, t)+b \theta^{d-1} R(\theta, t)-\theta^{d} R(\theta, t), \quad|\theta|<c(t),
$$

where $R(\theta, t)$ is the moment generating function of resolvent (3) for the random walk $\xi_{n}$, $n>0$. Comparing the coefficients for $\theta^{k}, k>0$, and putting $R_{m}(t)=0, m \leq 0$, we get

$$
Q_{k, d}^{t}=\left(\frac{Q_{1, d}^{t}}{R_{1}(t)}-b \delta_{1, d}\right) R_{k}(t)+b R_{k-d+1}(t)-R_{k-d}(t) .
$$

We look for a function $Q_{1, d}^{t}=\tilde{Q}_{1, d}^{t}$ for which solution (17) of the infinite system (16) is also a solution of the initial system. Substituting expression (17) into the initial system (15) we get by Lemma 2 that

$$
\frac{\tilde{Q}_{1, d}^{t}}{R_{1}(t)}-b \delta_{1, d}=b^{N} \frac{R_{N-d}(t)}{\hat{R}_{N}(b, t)} .
$$

Combining this expression and (17) we obtain

$$
Q_{k, d}^{t}=b^{N} \frac{R_{N-d}(t)}{\hat{R}_{N}(b, t)} R_{k}(t)+b R_{k-d+1}(t)-R_{k-d}(t), \quad k, d \in\{1, \ldots, N-1\} .
$$

Now put $\mathrm{P}\left[\xi_{0}=0\right]=1$, and let $k$ and $r$ be positive integers, $N=k+r$. Let

$$
\tau=\inf \left\{n>0: \xi_{n} \notin\{-r+1, \ldots, k-1\}\right\}
$$

be the first moment when the random walk $\xi_{n}, n>0$, exits the set

$$
\{-r+1, \ldots, k-1\} \text {. }
$$

Denote by

$$
\mathrm{P}_{(-r, k)}^{n}[i]=\mathrm{P}\left[\xi_{n}=i, \tau>n\right], \quad-r<i<k,
$$

the transient probability for the random walk $\xi_{n}$ on the interval $0 \leq n<\tau$. Since the random walk $\xi_{n}, n \geq 0$, is homogeneous, we find from (18) that

$$
\sum_{n=0}^{\infty} t^{n} \mathrm{P}_{(-r, k)}^{n}[i]=b^{N} \frac{R_{k}(t)}{\hat{R}_{N}(b, t)} R_{i+r}(t)+b R_{i+1}(t)-R_{i}(t), \quad-r<i<k .
$$

Since

we have

$$
\mathrm{P}[\tau>n]=\mathrm{P}\left[-r<\mu_{n}^{-}, \mu_{n}^{+}<k\right]
$$

whence Theorem 2 follows.

$$
\mathrm{P}_{(-r, k)}^{n}[i]=\mathrm{P}\left[-r<\mu_{n}^{-}, \xi_{n}=i, \mu_{n}^{+}<k\right], \quad-r<i<k,
$$




\section{A RANDOM WALK $\xi_{n}, n \geq 0$, AND TWO ABSORBING BOUNDARIES}

Fix a positive integer $N$ and consider a random sequence $X_{n} \in\{0,1, \ldots, N\}, n \geq 0$, whose one-step transient probabilities are given by

$$
k \rightarrow \begin{cases}i, & \mathrm{P}[\xi=i-k], i \in\{1, \ldots, N-1\} \\ 0, & \mathrm{P}[\xi \leq-k], \\ N, & \mathrm{P}[\xi \geq N-k]\end{cases}
$$

for all $k \in\{0, \ldots, N\}$.

The homogeneous (discrete time) Markov process $X_{n}, n \geq 0$, describes the evolution of the random walk $\xi_{n}, n \geq 0$, in the strip $0, \ldots, N$ with two reflecting boundaries with absorption (the upper boundary is at the level $N$, while the lower one is at the level 0 ).

If the process $X_{n}, n \geq 0$, reaches the boundary 0 at a moment $n_{0}$, it stays there for a random time $\nu(0)$ until the first positive jump of the random walk $\xi_{n-n_{0}}, n \geq n_{0}$, occurs. Then the process is "reflected" and appears at a state $i \in\{1, \ldots, N-1\}$ with probability $\mathrm{P}[\xi=i]$ (the probability of its appearing on the upper boundary is $\mathrm{P}[\xi \geq N]$ ). It is clear that $\nu(0)$ is a geometric random variable and

$$
\mathrm{P}[\nu(0)=n]=\mathrm{P}[\xi>0](\mathrm{P}[\xi \leq 0])^{n-1}, \quad n>0 .
$$

If the process $X_{n}, n \geq 0$, reaches the upper boundary $N$ at a moment $n_{N}$, it stays there for a random time $\nu(N)$ until the first negative jump of the random walk $\xi_{n-n_{N}}$, $n \geq n_{N}$, occurs. Then the process is "reflected" and appears at a state $i \in\{1, \ldots, N-1\}$ with probability $\mathrm{P}[\xi=i-N]$ (the probability of its appearing on the lower boundary is $\mathrm{P}[\xi \leq-N])$. It is clear that $\nu(N)$ is a geometric random variable and

$$
\mathrm{P}[\nu(N)=n]=\mathrm{P}[\xi<0](\mathrm{P}[\xi \geq 0])^{n-1}, \quad n>0 .
$$

Random walks with two reflecting boundaries are useful for queueing systems having a bounded number of waiting places as well as in storage and inventory theories, and in other applied probability fields.

4.1. Hitting the boundaries. Let $\mathrm{P}\left[X_{0}=k\right]=1$, and let

$$
\tau_{k}^{0}=\inf \left\{n>0: X_{n}=0\right\}, \quad k \in\{1, \ldots, N\},
$$

be the first time when the process $X_{n}, n \geq 0$, reaches the lower boundary 0 . Also let

$$
\tau_{k}^{N}=\inf \left\{n>0: X_{n}=N\right\}, \quad k \in\{0, \ldots, N-1\},
$$

be the first time when the process $X_{n}, n \geq 0$, reaches the upper boundary $N$.

Theorem 3. The moment generating functions of the random variables $\tau_{k}^{0}$ and $\tau_{k}^{N}$ and their means $\mathrm{E}\left[\tau_{k}^{0}\right]$ and $\mathrm{E}\left[\tau_{k}^{N}\right]$ satisfy

$$
\begin{gathered}
\mathrm{E}\left[t^{\tau_{k}^{0}}\right]=\frac{b^{N}}{1-b} \frac{1+(1-b)(1-t) S_{N-k}(t)}{b^{N} /(1-b)+(1-b)(1-t) \hat{S}_{N}(b, t)}, \\
\mathrm{E}\left[\tau_{k}^{0}\right]=(1-b)\left[b^{-N} \hat{R}_{N}(b)+S_{N-1}-S_{N-k}\right], \\
\sum_{n=0}^{\infty} t^{n} \mathrm{P}\left[\tau_{k}^{N}>n\right]=(1-b)\left\{\frac{\hat{R}_{N+1}(b, t)}{\hat{R}_{N+1}(b, t)-b \hat{R}_{N}(b, t)} R_{N-k}(t)-S_{N-k}(t)\right\}, \\
\mathrm{E}\left[\tau_{k}^{N}\right]=(1-b)\left\{\frac{\hat{R}_{N+1}(b)}{\hat{R}_{N+1}(b)-b \hat{R}_{N}(b)} R_{N-k}-S_{N-k}\right\},
\end{gathered}
$$


where

$$
\begin{gathered}
\hat{R}_{N}(b, t)=\sum_{i=N}^{\infty} b^{i} R_{i}(t), \quad \hat{R}_{N}(b)=\sum_{i=N}^{\infty} b^{i} R_{i}=\hat{R}_{N}(b, 1), \\
\hat{S}_{N}(b, t)=\sum_{i=N}^{\infty} b^{i} S_{i}(t), \quad S_{k}(t)=\sum_{i=1}^{k} R_{i}(t) .
\end{gathered}
$$

Proof of Theorem 3. Let $\mathrm{P}\left[X_{0}=N-k\right]=1$. Using the transient probabilities (19) of the process $X_{n}, n \geq 0$, we see that the moment generating function

$$
\tilde{\varphi}_{k}(t)=\mathrm{E}\left[t^{\tau_{N-k}^{0}}\right], \quad t \in(0,1), k \in\{0, \ldots, N-1\}
$$

satisfies the system of equations

$$
\begin{gathered}
\tilde{\varphi}_{k}(t)=t \mathrm{P}[\xi \leq k-N]+t \tilde{\varphi}_{0}(t) \mathrm{P}[\xi \geq k]+t \sum_{i=1}^{N-1} \tilde{\varphi}_{i}(t) p_{k-i}, \\
k \in\{0, \ldots, N-1\},
\end{gathered}
$$

by the full probability formula. System (20) is strongly regular, that is,

$$
t \mathrm{P}[\xi \geq k]+t \sum_{i=1}^{N-1} \mathrm{P}[\xi=k-i]=t \mathrm{P}[\xi>k-N]<1-t b^{N-1} \mathrm{E}\left[b^{\alpha}\right] .
$$

Therefore it has a unique solution (see [10]).

Using the transient probabilities (19) of the process $X_{n}, n \geq 0$, we prove that the moment generating function

$$
\tilde{\psi}_{k}(t)=\mathrm{E}\left[t^{\tau_{N-k}^{N}}\right], \quad k \in\{1, \ldots, N\}, t \in[0,1),
$$

satisfies the system of equations

$$
\tilde{\psi}_{k}(t)=t \mathrm{P}[\xi \geq k]+t \mathrm{P}[\xi \leq k-N] \tilde{\psi}_{N}(t)+t \sum_{i=1}^{N} \tilde{\psi}_{i}(t) p_{k-i}, \quad k \in\{1, \ldots, N\},
$$

by the full probability formula. The latter system is strongly regular, since

$$
t \mathrm{P}[\xi<k-N]+t \mathrm{P}[k-N \leq \xi \leq k-1]<t \mathrm{P}[\xi \leq N-1]<1-t \mathrm{E}\left[1-b^{\alpha-N} ; \alpha>N\right] .
$$

Thus it has a unique solution (see [10]).

Applying to systems (20) and (21) the method used in the preceding sections for solving corresponding systems of equations, we complete the proof of Theorem 3 .

4.2. Transient probabilities and ergodic distribution of the process $X_{n}, n \geq 0$. Let $N>0$ and let $\nu_{t}$ be a geometric random variable with parameter $t: \mathrm{P}\left[\nu_{t}=n\right]=$ $(1-t) t^{n}, n \geq 0, t \in[0,1)$.

Theorem 4. Let $\mathrm{P}\left[X_{0}=k\right]=1, k \in\{0, \ldots, N-1\}$. Then

$$
\begin{gathered}
\mathrm{P}\left[X_{\nu_{t}}=i\right]=\frac{b^{N}}{1-b} \frac{R_{i+1}(t)-R_{i}(t)}{\hat{R}_{N+1}(b, t)}\left[1+(1-b)(1-t) S_{N-k}(t)\right] \\
\quad+(1-t)\left[b R_{i-k+1}(t)-R_{i-k}(t)\right], \quad i \in\{0, \ldots, N-1\}, \\
\mathrm{P}\left[X_{\nu_{t}}=N\right]=\frac{\hat{R}_{N+1}(b, t)-b \hat{R}_{N}(b, t)}{(1-b) \hat{R}_{N+1}(b, t)}\left[1+(1-b)(1-t) S_{N-k}(t)\right]-(1-t) R_{N-k}(t) .
\end{gathered}
$$

Moreover there exists the ergodic distribution

$$
\Pi_{i}=\lim _{n \rightarrow \infty} \mathrm{P}\left[X_{n}=i\right], \quad i \in\{0, \ldots, N\},
$$


of the process $X_{n}, n \geq 0$, such that

$$
\Pi_{i}=\frac{b^{N+1}}{1-b} \frac{R_{i+1}-R_{i}}{\hat{R}_{N+1}(b)}, \quad i \in\{0, \ldots, N-1\}, \quad \Pi_{N}=\frac{\hat{R}_{N+1}(b)-b \hat{R}_{N}(b)}{(1-b) \hat{R}_{N+1}(b)} .
$$

Proof of Theorem 4. Let $\mathrm{P}\left[X_{0}=N-k\right]=1, k \in\{0, \ldots, N\}$. Denote by

$$
\mathrm{P}_{k r}(n)=\mathrm{P}\left[X_{n}=N-r\right], \quad r \in\{0, \ldots, N\},
$$

the transient probability of the process $X_{n}, n \geq 0$. By the full probability formula and (19), the functions

$$
\mathrm{P}_{k, r}^{t}=\sum_{n=0}^{\infty} t^{n} \mathrm{P}_{k r}(n), \quad t \in[0,1)
$$

satisfy the system

$$
\begin{gathered}
\mathrm{P}_{k, r}^{t}=\delta_{k, r}+t \mathrm{P}_{N, r}^{t} \mathrm{P}[\xi<k-N]+t \mathrm{P}_{0, r}^{t} \mathrm{P}[\xi \geq k]+t \sum_{i=1}^{N} \mathrm{P}_{i, r}^{t} p_{k-i}, \\
k, r \in\{0, \ldots, N\} .
\end{gathered}
$$

This problem is the most complicated among similar problems considered in this paper. Nevertheless the method used for solving the preceding two-boundary problems works in this case, too.

The functions $b \mathrm{P}_{k+1, r}^{t}, k \in\{-1, \ldots, N-1\}$, satisfy the following equations:

$$
b \mathrm{P}_{k+1, r}^{t}=b \delta_{k+1, r}+t b \mathrm{P}_{N, r}^{t} \mathrm{P}[\xi<k+1-N]+t b \mathrm{P}_{0, r}^{t} \mathrm{P}[\xi \geq k+1]+t b \sum_{i=1}^{N} \mathrm{P}_{i, r}^{t} p_{k+1-i} .
$$

Subtracting (22) from the latter equality we get that for $k \in\{0, \ldots, N-1\}$,

$$
b \mathrm{P}_{k+1, r}^{t}-\mathrm{P}_{k, r}^{t}=b \delta_{k+1, r}-\delta_{k, r}-t(1-b) \mathrm{P}_{0, r}^{t} \mathrm{P}[\alpha \geq k+1]-t(1-b) \sum_{i=1}^{k+1} \mathrm{P}_{i, r}^{t} a_{k+1-i}
$$

Put

$$
\mathrm{P}_{r}(\theta, t)=\sum_{k=0}^{\infty} \theta^{k} \mathrm{P}_{k, r}^{t}, \quad|\theta|<c(t) .
$$

Solving equations (23) by the moment generating function method we obtain that for all $k \geq 0$,

$$
\begin{gathered}
\mathrm{P}_{r}(\theta, t)=\frac{\mathrm{P}_{0, r}^{t}}{1-\theta}[1+(1-b)(1-t) R(\theta, t)]+b\left(1-\delta_{r, 0}\right) \theta^{r-1} R(\theta, t)-\theta^{r} R(\theta, t), \\
|\theta|<c(t) .
\end{gathered}
$$

Comparing the coefficients for $\theta^{k}, k \geq 0$, on both sides of this equality, we see that

$$
\begin{gathered}
\mathrm{P}_{k, r}^{t}=\mathrm{P}_{0, r}^{t}\left[1+(1-b)(1-t) S_{k}(t)\right]+b\left(1-\delta_{r, 0}\right) R_{k+1-r}(t)-R_{k-r}(t), \\
R_{m}(t)=0, \quad m \leq 0 .
\end{gathered}
$$

Now we find a function $\mathrm{P}_{0, r}^{*}(t)$ such that $\mathrm{P}_{k, r}^{t}$ defined by $(24)$ for $k \in\{0, \ldots, N\}$ is a solution of the initial system (22). Substituting the right-hand side of (24) into (22) and applying Lemma 2 we prove that

$$
\begin{gathered}
\mathrm{P}_{00}^{*}(t)=\frac{\hat{R}_{N+1}(b, t)-b \hat{R}_{N}(b, t)}{(1-b)(1-t) \hat{R}_{N+1}(b, t)}, \\
\mathrm{P}_{0 r}^{*}(t)=\frac{b^{N+1}}{1-b} \frac{R_{N+1-r}(t)-R_{N-r}(t)}{(1-t) \hat{R}_{N+1}(b, t)}, \quad r \in\{1, \ldots, N\},
\end{gathered}
$$


where

$$
\hat{R}_{N}(b, t)=\sum_{i=N}^{\infty} b^{i} R_{i}(t) .
$$

Substituting $\mathrm{P}_{0 r}^{*}(t)$ into (24) we find that for all $k, r \in\{0, \ldots, N\}$,

$$
\begin{aligned}
\mathrm{P}_{k, r}^{t}= & \frac{b^{N+1}}{1-b} \frac{R_{N+1-r}(t)-R_{N-r}(t)}{(1-t) \hat{R}_{N+1}(b, t)}\left[1+(1-b)(1-t) S_{k}(t)\right] \\
& +b R_{k+1-r}(t)-R_{k-r}(t), \quad r \in\{1, \ldots, N\}, \\
\mathrm{P}_{k, 0}^{t}= & \frac{\hat{R}_{N+1}(b, t)-b \hat{R}_{N}(b, t)}{(1-t)(1-b) \hat{R}_{N+1}(b, t)}\left[1+(1-b)(1-t) S_{k}(t)\right]-R_{k}(t) .
\end{aligned}
$$

Since

$$
\mathrm{P}\left[X_{\nu_{t}}=i\right]=(1-t) \mathrm{P}_{N-k, N-i}^{t}, \quad k, i \in\{0, \ldots, N\},
$$

the first two identities of Theorem 4 follow from (25).

The process $X_{n}, n \geq 0$, is a Markov chain with a finite set of states $\{0, \ldots, N\}$. If $\mathrm{P}[\alpha>1]>0$, then there exists $n_{0}$ such that $\min _{k, r} \mathrm{P}_{k, r}\left(n_{0}\right)>0$. Then (see [11]) there exists the ergodic distribution

$$
\Pi_{i}=\lim _{n \rightarrow \infty} \mathrm{P}\left[X_{n}=i\right], \quad i \in\{0, \ldots, N\} .
$$

To get the ergodic distribution in an explicit form, one needs to evaluate the limits

$$
\Pi_{i}=\lim _{t \rightarrow 1}(1-t) \mathrm{P}_{k, N-i}^{t}, \quad i \in\{0, \ldots, N\} .
$$

Multiplying equalities $(25)$ by $(1-t)$ and passing to the limits as $t \rightarrow 1$, we get by changing $r \rightarrow N-i$ that

$$
\Pi_{i}=\frac{b^{N+1}}{1-b} \frac{R_{i+1}-R_{i}}{\hat{R}_{N+1}(b)}, \quad i \in\{0, \ldots, N-1\}, \quad \Pi_{N}=\frac{\hat{R}_{N+1}(b)-b \hat{R}_{N}(b)}{(1-b) \hat{R}_{N+1}(b)}
$$

where $R_{k}, k>0$, is potential (4) of the random walk $\xi_{n}, n \geq 0$. Theorem 4 is proved.

Remark 1. Putting $b=0$ in our theorems and corollaries we obtain the corresponding results for a lower semicontinuous random walk $\xi_{n}, n \geq 0$, whose increments have the moment generating function

$$
\sum_{n=0}^{\infty} t^{n} \mathrm{E}\left[\theta^{\xi_{n}}\right]=\left\{1-\frac{t}{\theta} \mathrm{E}\left[\theta^{\alpha}\right]\right\}^{-1}, \quad|\theta|=1, t \in(0,1) .
$$

\section{BIBLIOGRAPHY}

1. L. Takács, Combinatorial Methods in the Theory of Stochastic Processes, Wiley, New YorkLondon-Sydney, 1967. MR 36:947

2. V. S. Korolyuk, Boundary Problems for Compound Poisson Processes, "Naukova Dumka", Kiev, 1975. (Russian) MR 53:6753

3. V. N. Suprun and V. M. Shurenkov, On the resolvent of a process with independent increments terminating at the moment when it hits the negative real semiaxis, Studies in the Theory of Stochastic Processes, Institute of Mathematics, Academy of Sciences of UkrSSR, Kiev, 1975, pp. 170-174. (Russian) MR 55:13583

4. V. N. Suprun, Ruin problem and the resolvent of a terminating process with independent increments, Ukr. Mat. Zh. 28 (1976), no. 1, 53-61; English transl. in Ukr. Math. J. 28 (1976). MR 55:1497

5. D. V. Gusak, Boundary Problems for Processes with Independent Increments Defined on Finite Markov Chains and for Semi-Markov Processes, Institute of Mathematics, National Academy of Sciences of Ukraine, Kyiv, 1998. (Ukrainian) MR 2000m:60050

6. - On a generalized semicontinuous integer-valued Poisson process with reflection, Teor. Imovirnost. ta Matem. Statist. 59 (1998), 40-46; English transl. in Theor. Probability and Math. Statist. 59 (1999), 41-46. 
7. E. A. Pecherskiŭ, Some identities related to the exit of a random walk from a segment and a semisegment, Teor. Veroyatnost. i Primenen. 19 (1974), no. 1, 104-119; English transl. in Theory Probab. Appl. 19 (1975), no. 1. MR 49:6366

8. M. V. Kartashov, On ruin probabilities for a risk process with bounded reserves, Teor. Imovirnost. ta Matem. Statist. 60 (1999), 46-58; English transl. in Theor. Probability and Math. Statist. 60 (2000), 53-65.

9. F. Spitzer, Principles of Random Walk, Springer-Verlag, Berlin-New York, 1976. MR 52:9383

10. L. V. Kantorovich and V. I. Krylov, Approximate Methods of Higher Analysis, "Nauka", Moscow-Leningrad, 1962; English transl., Interscience Publishers, Inc./P. Noordhoff Ltd., New York/Gröningen, 1958. MR 27:4338

11. A. N. Shiryaev, Probability, "Nauka", Moscow, 1980; English transl., Springer-Verlag, New York, 1984. MR 82d:60002

Faculty for Mechanics and Mathematics, Kyiv National Taras Shevchenko University, Academician Glushiov Avenue 6, Kyiv, Ukraine

E-mail address: t_thys@ukr.net

Received 27/SEP/2002

Translated by V. SEMENOV 